\title{
A new approach to slice regularity on real algebras
}

\author{
Riccardo Ghiloni and Alessandro Perotti
}

\begin{abstract}
We expose the main results of a theory of slice regular functions on a real alternative algebra $A$, based on a well-known Fueter's construction. Our general theory includes the theory of slice regular functions of a quaternionic or octonionic variable and the theory of slice monogenic functions of a Clifford variable. Our approach permits to extend the range of these function theories and to obtain new results. In particular, we show that a fundamental theorem of algebra with multiplicities holds for an ample class of polynomials with coefficients in $A$. We give several examples to illustrate some interesting aspects of the theory.
\end{abstract}

Mathematics Subject Classification (2000). Primary 30C15; Secondary 30G35, 32A30.

Keywords. Functions of a hypercomplex variable, Quaternions, Octonions, Clifford algebras, Fundamental theorem of algebra.

\section{Introduction}

In this survey paper, we propose a new approach to the concepts of "slice regularity" for functions of one quaternionic, octonionic or Clifford variable which have been recently introduced by Gentili and Struppa in $[17,18]$ and by Colombo, Sabadini and Struppa in [8].

Actually, the starting point for our approach is not new: it dates back to a paper of Rudolf Fueter [12], in which he proposed a simple method, which is now known as Fueter's Theorem, to generate quaternionic regular functions (cf. [35] and [25] for the theory of Fueter regular functions) by means of complex holomorphic functions. Given a holomorphic "stem function"

$$
F(z)=u(\alpha, \beta)+i v(\alpha, \beta) \quad(z=\alpha+i \beta \text { complex, } u, v \text { real-valued })
$$

Partially supported by MIUR (Project "Proprietà geometriche delle varietà reali e complesse") and GNSAGA of INdAM. 
in the upper complex half-plane, real-valued on $\mathbb{R}$, the formula

$$
f(q):=u\left(q_{0},|\operatorname{Im}(q)|\right)+\frac{\operatorname{Im}(q)}{|\operatorname{Im}(q)|} v\left(q_{0},|\operatorname{Im}(q)|\right)
$$

(with $q=q_{0}+q_{1} i+q_{2} j+q_{3} k \in \mathbb{H}, \operatorname{Im}(q)=q_{1} i+q_{2} j+q_{3} k$ ) defines a function on $\mathbb{H}$, whose Laplacian is Fueter regular. Fueter's construction was later extended to higher dimensions by Sce [32], Qian [30] and Sommen [34] in the setting of octonionic and Clifford analysis.

We extend Fueter's construction in order to develop a theory of slice regular functions on a real alternative algebra $A$ with a fixed antiinvolution. These functions will be obtained by taking $A$-valued components $u, v$ of the stem function $F$. The domains on which slice regular functions can be defined are open subsets of what we call the quadratic cone of the algebra. This cone is the whole algebra only in the case in which $A$ is a real division algebra (i.e. the complex numbers, the quaternions $\mathbb{H}$ or the octonions $(\mathbb{D})$. We refer the reader to the article [22] for complete proofs of the main results stated in the present paper.

If $A$ is the algebra of quaternions, we get the theory of slice regular (or Cullen regular) functions of a quaternionic variable introduced by Gentili and Struppa $[17,18]$. If $A$ is the algebra of octonions, we obtain the corresponding theory of regular functions already considered in [16, 20,23]. If $A$ is the Clifford algebra $C l_{0, n}=\mathbb{R}_{n}$, the quadratic cone is a real algebraic (proper for $n \geq 3$ ) subset of $\mathbb{R}_{n}$, containing the subspace of paravectors. By restricting the Clifford variables to the paravectors, we get the theory of slice monogenic functions introduced by Colombo, Sabadini and Struppa in [8].

In Section 2, we define the normal cone and the quadratic cone of an algebra $A$ and prove that the quadratic cone is a union of complex planes of $A$. This property is the starting point for the extension of Fueter's construction. We compute the cones for some relevant algebras. In particular, we show that the quadratic cone can be a semi-algebraic set (e.g. in Clifford algebras with non-definite signature) and depends on the antiinvolution chosen in $A$. We also find the dimensions of the cones.

In Section 3, we introduce complex intrinsic functions with values in the complexified algebra $A \otimes_{\mathbb{R}} \mathbb{C}$ and use them as stem functions to generate $A$-valued (left) slice functions. This approach does not require the holomorphy of the stem function. Moreover, slice functions can be defined also on domains which do not intersect the real axis of $A$.

In Section 4, we restrict our attention to slice functions with holomorphic stem function, what we call (left) slice regular functions on $A$. These functions forms a real vector space that is not closed w.r.t. the pointwise product in $A$. The pointwise product for stem functions induces a natural product on slice functions (cf. Section 5), that generalizes the usual product of polynomials and power series.

In Section 6, we recall some properties of the zero set of slice functions. We restrict our attention to admissible slice regular functions, which preserve many relevant properties of classical holomorphic functions. We generalize a structure 
theorem for the zero set proved by Pogorui and Shapiro [29] for quaternionic polynomials and by Gentili and Stoppato [15] for quaternionic power series. A Remainder Theorem (Theorem 11) gives us the possibility to define a notion of multiplicity for the zeros of an admissible slice regular function.

Polynomials with right coefficients in $A$ are slice regular functions on the quadratic cone. We obtain a version of the fundamental theorem of algebra for slice regular admissible polynomials. This theorem was proved for quaternionic polynomials by Eilenberg and Niven [10, 28] and for octonionic polynomials by Jou [26]. See also [11, pp. 308ff] for a topological proof of the theorem valid for a class of real algebras including $\mathbb{C}, \mathbb{H}$ and $\mathbb{O}$, and [36], [31] and [21] for other proofs. Gordon and Motzkin [24] proved, for polynomials on a (associative) division ring, that the number of conjugacy classes containing zeros of $p$ cannot be greater than the degree $m$ of $p$. This estimate was improved on $\mathbb{H}$ by Pogorui and Shapiro [29]: if $p$ has $s$ spherical zeros and $l$ non-spherical zeros, then $2 s+l \leq m$. Gentili and Struppa [19] showed that, using the right definition of multiplicity, the number of zeros of $p$ equals the degree of the polynomial. In [23], this strong form was generalized to the octonions. Recently, Colombo, Sabadini and Struppa [8, 9] and Yang and Qian [37] proved some results on the structure of the set of zeros of a polynomial with paravector coefficients in a Clifford algebra.

We obtain a strong form of the fundamental theorem of algebra (Theorem 14), which contains and generalizes the above results. We show that the sum of the multiplicities of the zeros of a slice regular admissible polynomial is equal to its degree. The last section contains several examples that illustrate the relevance of the quadratic cone and of the condition of admissibility for the algebraic and topological properties of the zero set of a polynomial. We see that the quadratic cone is sufficiently large to contain the "right" number of (isolated or spherical) zeros, and sufficiently small to exclude "wild" sets of zeros of the polynomial. Outside the quadratic cone, an admissible polynomial can have infinite non-spherical zeros. If the polynomial is not admissible, its zero set loses its regular structure.

\section{The quadratic cone of a real alternative algebra}

Let $A$ be a finite-dimensional alternative real algebra with a unity, of dimension $d>1$. We will identify the field of real numbers with the subalgebra of $A$ generated by the unity. In a real algebra, we can consider the imaginary space consisting of all non-real elements whose square is real.

Definition 1. Let $\operatorname{Im}(A):=\left\{x \in A \mid x^{2} \in \mathbb{R}, x \notin \mathbb{R} \backslash\{0\}\right\}$. The elements of $\operatorname{Im}(A)$ are called purely imaginary elements of $A$.

In general, the imaginary space $\operatorname{Im}(A)$ is not a vector subspace of $A$. In what follows, we will assume that on $A$ an antiinvolution is fixed. It is a linear map $x \mapsto x^{c}$ of $A$ into $A$ satisfying the following properties:

$$
\left(x^{c}\right)^{c}=x \quad \forall x \in A, \quad(x y)^{c}=y^{c} x^{c} \quad \forall x, y \in A, \quad x^{c}=x \quad \text { for every real } x .
$$


Definition 2. For every element $x$ of $A$, the trace of $x$ is $t(x):=x+x^{c} \in A$ and the (squared) norm of $x$ is $n(x):=x x^{c} \in A$.

Definition 3. We call normal cone of the algebra $A$ the subset

$$
\mathcal{N}_{A}:=\{0\} \cup\left\{x \in A \mid n(x)=n\left(x^{c}\right) \in \mathbb{R} \backslash\{0\}\right\},
$$

and quadratic cone of $A$ the set

$$
\mathcal{Q}_{A}:=\mathbb{R} \cup\left\{x \in A \mid t(x) \in \mathbb{R}, n(x) \in \mathbb{R}, 4 n(x)>t(x)^{2}\right\} .
$$

We also set $\mathbb{S}_{A}:=\left\{J \in \mathcal{Q}_{A} \mid J^{2}=-1\right\} \subseteq \operatorname{Im}(A)$. Elements of $\mathbb{S}_{A}$ will be called square roots of -1 in the algebra $A$. For every $J \in \mathbb{S}_{A}$, we will denote by $\mathbb{C}_{J}:=\langle 1, J\rangle \simeq \mathbb{C}$ the subalgebra of $A$ generated by $J$.

Proposition 1. Let $A$ be an alternative real algebra with a fixed antiinvolution $x \mapsto x^{c}$. The following statements hold.

(1) Every $x \in \mathcal{Q}_{A}$ satisfies the real quadratic equation $x^{2}-x t(x)+n(x)=0$.

(2) $x \in \mathcal{Q}_{A}$ is equivalent to $x^{c} \in \mathcal{Q}_{A}$. Moreover, $\mathcal{Q}_{A} \subseteq \mathcal{N}_{A}$.

(3) Every nonzero $x \in \mathcal{N}_{A}$ is invertible: $x^{-1}=n(x)^{-1} x^{c}$.

(4) $J^{c}=-J$ for every $J \in \mathbb{S}_{A}$, i.e. $t(J)=0, n(J)=1$.

(5) $\mathcal{Q}_{A}=A$ if and only if $A$ is isomorphic to one of the division algebras $\mathbb{C}, \mathbb{H}$ or $\mathbb{O}$ with the usual conjugation mapping.

(6) For every $x \in \mathcal{Q}_{A}$, there exist uniquely determined elements $x_{0} \in \mathbb{R}, y \in$ $\operatorname{Im}(A) \cap \mathcal{Q}_{A}$, with $t(y)=0$, such that $x=x_{0}+y$.

(7) $\mathcal{Q}_{A}=\bigcup_{J \in \mathbb{S}_{A}} \mathbb{C}_{J}$ and $\mathbb{C}_{I} \cap \mathbb{C}_{J}=\mathbb{R}$ for every $I, J \in \mathbb{S}_{A}, I \neq \pm J$.

Using the notation of the above proposition, for every $x \in \mathcal{Q}_{A}$, we set $\operatorname{Re}(x):=x_{0}=\frac{x+x^{c}}{2}, \operatorname{Im}(x):=y=\frac{x-x^{c}}{2}$.

As shown by the examples below, the quadratic cone $\mathcal{Q}_{A}$ needs not be a subalgebra or a subspace of $A$.

Examples 1. (1) Let $A$ be the algebra $\mathbb{H}$ of the quaternions or the algebra $\mathbb{O}$ of the octonions. Let $x^{c}=\bar{x}$ be the usual conjugation mapping. Then $\operatorname{Im}(A)$ is a subspace, $A=\mathbb{R} \oplus \operatorname{Im}(A)$ and $\mathcal{Q}_{\mathbb{H}}=N_{\mathbb{H}}=\mathbb{H}, \mathcal{Q}_{\mathbb{O}}=N_{\mathbb{O}}=\mathbb{O}$. In these cases, $\mathbb{S}_{\mathbb{H}}$ is a two-dimensional sphere and $\mathbb{S}_{\mathbb{O}}$ is a six-dimensional sphere.

(2) Let $A$ be the real Clifford algebra $C l_{p, q}=\mathbb{R}_{p, q}$, with the conjugation $x^{c}=\left([x]_{0}+[x]_{1}+[x]_{2}+[x]_{3}+[x]_{4}+\cdots\right)^{c}=[x]_{0}-[x]_{1}-[x]_{2}+[x]_{3}+[x]_{4}-\cdots$, where $[x]_{k}$ denotes the $k$-vector component of $x$ in $\mathbb{R}_{p, q}$ (cf. for example $[7, \S 4.1]$ or $[25, \S 3.2])$. Let $n:=p+q$. An element $x$ of $\mathbb{R}_{p, q}$ can be represented in the form $x=\sum_{K} x_{K} e_{K}$, with $K=\left(i_{1}, \ldots, i_{k}\right)$ an increasing multiindex of length $k$, $0 \leq k \leq n, e_{K}=e_{i_{1}} \cdots e_{i_{k}}, e_{\emptyset}=1, x_{K} \in \mathbb{R}, x_{\emptyset}=x_{0}, e_{1}, \ldots, e_{n}$ basis elements.

Let $\mathbb{R}_{n}:=\mathbb{R}_{0, n}$. In this case, the quadratic cone $\mathcal{Q}_{n}:=\mathcal{Q}_{\mathbb{R}_{n}}$ is the real algebraic (proper for $n \geq 3$ ) subset of $\mathbb{R}_{n}$ defined in terms of the euclidean scalar product $x \cdot y$ by the equations

$$
x_{K}=0, x \cdot\left(x e_{K}\right)=0 \text { for every } e_{K} \neq 1 \text { such that } e_{K}^{2}=1 .
$$


The subspace of paravectors $\mathbb{R}^{n+1}:=\left\{x \in \mathbb{R}_{n} \mid[x]_{k}=0\right.$ for every $\left.k>1\right\}$ is contained in $\mathcal{Q}_{n}$. For $x \in \mathbb{R}^{n+1}, t(x)=2 x_{0} \in \mathbb{R}$ and $n(x)=|x|^{2} \geq 0$ (the euclidean norm). The $(n-1)$-dimensional sphere $\mathbb{S}=\left\{x=x_{1} e_{1}+\cdots x_{n} e_{n} \in\right.$ $\left.\mathbb{R}^{n+1} \mid x_{1}^{2}+\cdots+x_{n}^{2}=1\right\}$ of unit 1 -vectors is (properly) contained in $\mathbb{S}_{\mathbb{R}_{n}}$. The normal cone $\mathcal{N}_{n}:=\mathcal{N}_{\mathbb{R}_{n}}$ contains also the Clifford group $\Gamma_{n}$ and its subgroups $\operatorname{Pin}(n)$ and $\operatorname{Spin}(n)$. If $n \geq 3, \operatorname{Im}(A)$ is not a subspace of $A$.

(3) The case of $\mathbb{R}_{3}$. A direct computation shows that

$$
\mathcal{N}_{3}=\left\{x \in \mathbb{R}_{3} \mid x_{0} x_{123}+x_{2} x_{13}-x_{1} x_{23}-x_{3} x_{12}=0\right\}
$$

and the quadratic cone is the 6-dimensional real algebraic set

$$
\mathcal{Q}_{3}=\left\{x \in \mathbb{R}_{3} \mid x_{123}=0, x_{2} x_{13}-x_{1} x_{23}-x_{3} x_{12}=0\right\} .
$$

Finally, $\mathbb{S}_{\mathbb{R}_{3}}=\left\{x \in \mathcal{Q}_{3} \mid x_{0}=0, \sum_{i} x_{i}^{2}+\sum_{j, k} x_{j k}^{2}=1\right\} \subset \operatorname{Im}\left(\mathbb{R}_{3}\right) \cap \mathcal{Q}_{3}$ is the intersection of a 5 -sphere with the hypersurface $x_{2} x_{13}-x_{1} x_{23}-x_{3} x_{12}=0$.

(4) The case of $\mathbb{R}_{4}$. The normal cone $\mathcal{N}_{4}$ is the 11 -dimensional real algebraic set with equations

$$
\begin{aligned}
& x_{1} x_{1234}+x_{124} x_{13}-x_{12} x_{134}-x_{123} x_{14}=0, x_{2} x_{1234}+x_{124} x_{23}-x_{12} x_{234}-x_{123} x_{24}=0, \\
& x_{3} x_{1234}+x_{134} x_{23}-x_{13} x_{234}-x_{123} x_{34}=0, x_{4} x_{1234}-x_{14} x_{234}+x_{134} x_{24}-x_{124} x_{34}=0, \\
& x_{134} x_{2}-x_{1} x_{234}-x_{124} x_{3}+x_{123} x_{4}=0, x_{0} x_{1234}-x_{14} x_{23}+x_{13} x_{24}-x_{12} x_{34}=0, \\
& x_{0} x_{234}+x_{3} x_{24}-x_{2} x_{34}-x_{23} x_{4}=0, x_{0} x_{134}+x_{3} x_{14}-x_{1} x_{34}-x_{13} x_{4}=0, \\
& x_{0} x_{124}+x_{2} x_{14}-x_{1} x_{24}-x_{12} x_{4}=0, x_{0} x_{123}+x_{2} x_{13}-x_{1} x_{23}-x_{12} x_{3}=0,
\end{aligned}
$$

while the quadratic cone $\mathcal{Q}_{4}$ is the 8 -dimensional real algebraic set defined by

$$
\begin{aligned}
& x_{14} x_{23}-x_{13} x_{24}+x_{12} x_{34}=x_{3} x_{24}-x_{2} x_{34}-x_{23} x_{4}=x_{3} x_{14}-x_{1} x_{34}-x_{13} x_{4}=0, \\
& x_{2} x_{14}-x_{1} x_{24}-x_{12} x_{4}=x_{2} x_{13}-x_{1} x_{23}-x_{12} x_{3}=x_{123}=x_{124}=x_{134}=x_{234}=x_{1234}=0 .
\end{aligned}
$$

(5) Let $A=\mathbb{R}_{1,2}$. Then $\mathcal{N}_{1,2}$ is the 7 -dimensional real semi-algebraic set

$$
\left\{x \in \mathbb{R}_{1,2} \mid x_{0} x_{123}+x_{2} x_{13}-x_{1} x_{23}-x_{3} x_{12}=0, x_{0}^{2}+x_{2}^{2}+x_{3}^{2}+x_{23}^{2} \neq x_{1}^{2}+x_{12}^{2}+x_{13}^{2}+x_{123}^{2}\right\} \cup\{0\}
$$

and the quadratic cone $\mathcal{Q}_{1,2}$ is the 6 -dimensional real semi-algebraic set

$$
\begin{gathered}
\mathcal{Q}_{1,2}=\mathbb{R} \cup\left\{x \in \mathbb{R}_{1,2} \mid x_{123}=0, x_{2} x_{13}-x_{1} x_{23}-x_{3} x_{12}=0,\right. \\
\left.x_{2}^{2}+x_{3}^{2}+x_{23}^{2}>x_{1}^{2}+x_{12}^{2}+x_{13}^{2}\right\} .
\end{gathered}
$$

(6) The quadratic cone and the normal cone depend on the antiinvolution chosen in the algebra. For example, in the Clifford algebras $\mathbb{R}_{n}$, it is possible to take the reversion $x \mapsto x^{*}$ in place of the conjugation. The reversion is defined by $x^{*}=\left([x]_{0}+[x]_{1}+[x]_{2}+[x]_{3}+[x]_{4}+\cdots\right)^{*}=[x]_{0}+[x]_{1}-[x]_{2}-[x]_{3}+[x]_{4}-\cdots$.

In $\mathbb{R}_{2} \simeq \mathbb{H}$, we get the normal and quadratic cones

$\mathcal{N}_{2}^{*}=\left\{x \mid x_{1}=x_{2}=0\right\} \cup\left\{x \mid x_{0}=x_{12}=0\right\}, \mathcal{Q}_{2}^{*}=\left\{x \in \mathbb{R}_{2} \mid x_{1}=x_{2}=0\right\} \simeq \mathbb{C}$.

Here $\mathbb{S}_{A}=\left\{ \pm e_{12}\right\}$. 
In $\mathbb{R}_{3}$ the normal cone (w.r.t. reversion) is the 5 -dimensional real semialgebraic set

$$
\begin{gathered}
\mathcal{N}_{3}^{*}=\mathbb{R} \cup\left\{x \mid x_{2} x_{12}+x_{3} x_{13}=0, x_{1} x_{13}+x_{2} x_{23}=0, x_{1} x_{12}-x_{23} x_{3}=0,\right. \\
x_{0} x_{1}+x_{123} x_{23}=0, x_{0} x_{2}-x_{123} x_{13}=0, x_{0} x_{3}+x_{12} x_{123}=0, \\
\left.x_{0}^{2}+x_{12}^{2}+x_{13}^{2}+x_{23}^{2} \neq x_{1}^{2}+x_{2}^{2}+x_{3}^{2}+x_{123}^{2}\right\}
\end{gathered}
$$

and the quadratic cone is the 4 -dimensional plane

$$
\mathcal{Q}_{3}^{*}=\left\{x \mid x_{1}=x_{2}=x_{3}=x_{123}=0\right\}=\left\langle 1, e_{12}, e_{13}, e_{23}\right\rangle \simeq \mathbb{H} .
$$

\section{Slice functions}

Let $A_{\mathbb{C}}=A \otimes_{\mathbb{R}} \mathbb{C}$ be the complexification of $A$. We will use the representation

$$
A_{\mathbb{C}}=\{w=x+i y \mid x, y \in A\} \quad\left(i^{2}=-1\right) .
$$

$A_{\mathbb{C}}$ is an alternative complex algebra with a unity w.r.t. the product:

$$
(x+i y)\left(x^{\prime}+i y^{\prime}\right)=x x^{\prime}-y y^{\prime}+i\left(x y^{\prime}+y x^{\prime}\right) .
$$

The algebra $A$ can be identified with the real subalgebra $A^{\prime}:=\{w=x+i y \mid y=0\}$ of $A_{\mathbb{C}}$. In $A_{\mathbb{C}}$ two commuting operators are defined: the complex-linear antiinvolution $w \mapsto w^{c}=(x+i y)^{c}=x^{c}+i y^{c}$ and the complex conjugation defined by $\bar{w}=\overline{x+i y}=x-i y$.

Definition 4. Let $D \subseteq \mathbb{C}$ be an open subset. If a function $F: D \rightarrow A_{\mathbb{C}}$ is complex intrinsic, i.e. it satisfies the condition $F(\bar{z})=\overline{F(z)}$ for every $z \in D$ such that $\bar{z} \in D$, then $F$ is called an $A$-stem function on $D$.

In the preceding definition, there is no restriction to assume that $D$ is symmetric w.r.t. the real axis, i.e. $D=\operatorname{conj}(D):=\{z \in \mathbb{C} \mid \bar{z} \in D\}$.

Remarks 1. (1) A function $F$ is an $A$-stem function if and only if the $A$-valued components $F_{1}, F_{2}$ of $F=F_{1}+i F_{2}$ form an even-odd pair w.r.t. the imaginary part of $z$.

(2) By means of a basis $\mathcal{B}=\left\{u_{k}\right\}_{k=1, \ldots, d}$ of $A, F$ can be identified with a complex intrinsic curve in $\mathbb{C}^{d}$.

Given an open subset $D$ of $\mathbb{C}$, let $\Omega_{D}$ be the relatively open subset of $\mathcal{Q}_{A}$ obtained by the action on $D$ of the square roots of -1 :

$$
\Omega_{D}:=\left\{x=\alpha+\beta J \in \mathbb{C}_{J} \mid \alpha, \beta \in \mathbb{R}, \alpha+i \beta \in D, J \in \mathbb{S}_{A}\right\} .
$$

Sets of this type will be called circular sets in $A$.

Definition 5. Any stem function $F: D \rightarrow A_{\mathbb{C}}$ induces a left slice function $f=$ $\mathcal{I}(F): \Omega_{D} \rightarrow A$. If $x=\alpha+\beta J \in D_{J}:=\Omega_{D} \cap \mathbb{C}_{J}$, we set

$$
f(x):=F_{1}(z)+J F_{2}(z) \quad(z=\alpha+i \beta) .
$$


There is an analogous definition for right slice functions when $J$ is placed on the right of $F_{2}(z)$. In what follows, the term slice functions will always mean left slice functions. We will denote the real vector space of (left) slice functions on $\Omega_{D}$ by $\mathcal{S}\left(\Omega_{D}\right)$.

Examples 2. Assume $A=\mathbb{H}$ or $A=\mathbb{O}$, with the usual conjugation mapping.

(1) For any element $a \in A, F(z):=z^{n} a=\operatorname{Re}\left(z^{n}\right) a+i\left(\operatorname{Im}\left(z^{n}\right) a\right)$ induces the monomial $f(x)=x^{n} a \in \mathcal{S}(A)$.

(2) By linearity, we get all the standard polynomials $p(x)=\sum_{j=0}^{n} x^{j} a_{j}$ with right quaternionic or octonionic coefficients. More generally, every convergent power series $\sum_{j} x^{j} a_{j}$ is a slice function on an open ball of $A$ centered in the origin with (possibly infinite) radius.

The above examples generalize to standard polynomials in $x=\mathcal{I}(z)$ and $x^{c}=\mathcal{I}(\bar{z})$ with coefficients in $A$. The domain of slice polynomial functions or series must be restricted to subsets of the quadratic cone.

For an element $J \in \mathbb{S}_{A}$, let $\mathbb{C}_{J}^{+}$denote the upper half plane $\mathbb{C}_{J}^{+}=\{x=$ $\alpha+\beta J \in A \mid \beta \geq 0\}$.

Proposition 2. Let $J, K \in \mathbb{S}_{A}$ with $J-K$ invertible. Every slice function $f \in \mathcal{S}\left(\Omega_{D}\right)$ is uniquely determined by its values on the two distinct half planes $\mathbb{C}_{J}^{+}$and $\mathbb{C}_{K}^{+}$. Moreover, the following representation formula holds:

$$
f(x)=(I-K)\left((J-K)^{-1} f(\alpha+\beta J)\right)-(I-J)\left((J-K)^{-1} f(\alpha+\beta K)\right)
$$

for every $I \in \mathbb{S}_{A}$ and for every $x=\alpha+\beta I \in D_{I}=\Omega_{D} \cap \mathbb{C}_{I}$.

Representation formulas for quaternionic Cullen regular functions appeared in $[3,4]$, and for slice monogenic functions of a Clifford variable in $[6,5]$.

Definition 6. Let $f \in \mathcal{S}\left(\Omega_{D}\right)$ be a slice function.

The spherical value of $f$ in $x \in \Omega_{D}$ is $v_{s} f(x):=\frac{1}{2}\left(f(x)+f\left(x^{c}\right)\right)$.

The spherical derivative of $f$ in $x \in \Omega_{D} \backslash \mathbb{R}$ is $\partial_{s} f(x):=\frac{1}{2} \operatorname{Im}(x)^{-1}\left(f(x)-f\left(x^{c}\right)\right)$.

In this way, we get two $A$-valued slice functions associated with $f$, constant on every "sphere" $\mathbb{S}_{x}:=\left\{y \in \mathcal{Q}_{A} \mid y=\alpha+\beta I, I \in \mathbb{S}_{A}\right\}\left(x=\alpha+\beta J, J \in \mathbb{S}_{A}\right)$ such that $\partial_{s} f(x)=0$ if and only if $f$ is constant on $\mathbb{S}_{x}$. In this case, $f$ has value $v_{s} f(x)$ on $\mathbb{S}_{x}$. If $\Omega_{D} \cap \mathbb{R} \neq \emptyset$, under mild regularity conditions on $F$, we get that $\partial_{s} f$ can be continuously extended as a slice function on $\Omega_{D}$. By definition, the following identity holds for every $x \in \Omega_{D}: f(x)=v_{s} f(x)+\operatorname{Im}(x) \partial_{s} f(x)$.

Proposition 3. Let $f=\mathcal{I}(F) \in \mathcal{S}\left(\Omega_{D}\right)$ be a slice function. Then the following statements hold:

(1) If $F \in C^{0}(D)$, then $f \in C^{0}\left(\Omega_{D}\right)$.

(2) If $F \in C^{2 s+1}(D)$ for a positive integer $s$, then $f$ is of class $C^{s}\left(\Omega_{D}\right)$. As a consequence, if $F \in C^{\infty}(D)$, then $f$ is of class $C^{\infty}\left(\Omega_{D}\right)$.

(3) If $F \in C^{\omega}(D)$, then $f$ is of class $C^{\omega}\left(\Omega_{D}\right)$. 
We will denote by $\mathcal{S}^{1}\left(\Omega_{D}\right):=\left\{f=\mathcal{I}(F) \in \mathcal{S}\left(\Omega_{D}\right) \mid F \in C^{1}(D)\right\}$ the real vector space of slice functions with stem function of class $C^{1}$.

Definition 7. Let $f=\mathcal{I}(F) \in \mathcal{S}^{1}\left(\Omega_{D}\right)$. We set

$$
\frac{\partial f}{\partial x}:=\mathcal{I}\left(\frac{\partial F}{\partial z}\right), \quad \frac{\partial f}{\partial x^{c}}:=\mathcal{I}\left(\frac{\partial F}{\partial \bar{z}}\right) .
$$

These functions are continuous slice functions on $\Omega_{D}$.

\section{Slice regular functions}

Left multiplication by $i$ defines a complex structure on $A_{\mathbb{C}}$. With respect to this structure, a $C^{1}$ function $F=F_{1}+i F_{2}: D \rightarrow A_{\mathbb{C}}$ is holomorphic if and only if its components $F_{1}, F_{2}$ satisfy the Cauchy-Riemann equations.

Definition 8. A (left) slice function $f \in \mathcal{S}^{1}\left(\Omega_{D}\right)$ is (left) slice regular if its stem function $F$ is holomorphic. We will denote the real vector space of slice regular functions on $\Omega_{D}$ by $\mathcal{S R}\left(\Omega_{D}\right):=\left\{f \in \mathcal{S}^{1}\left(\Omega_{D}\right) \mid f=\mathcal{I}(F), F: D \rightarrow A_{\mathbb{C}}\right.$ holomorphic $\}$.

Polynomials with right coefficients in $A$ can be considered as slice regular functions on the quadratic cone. Assume that on $A$ is defined a positive scalar product $x \cdot y$ whose associated norm satisfies an inequality $|x y| \leq C|x||y|(C>0)$ and such that $|x|^{2}=n(x)$ for every $x \in \mathcal{Q}_{A}$. In this case we can consider also convergent power series $\sum_{k} x^{k} a_{k}$ as slice regular functions on the intersection of the quadratic cone with a ball centered in the origin. See for example $[25, \S 4.2]$ for the quaternionic and Clifford algebra cases, where we can take as product $x \cdot y$ the euclidean product in $\mathbb{R}^{4}$ or $\mathbb{R}^{2^{n}}$, respectively.

Proposition 4. Let $f=\mathcal{I}(F) \in \mathcal{S}^{1}\left(\Omega_{D}\right)$. Then $f$ is slice regular on $\Omega_{D}$ if and only if the restriction $f_{J}:=f_{\mid \mathbb{C}_{J} \cap \Omega_{D}}: D_{J}=\mathbb{C}_{J} \cap \Omega_{D} \rightarrow A$ is holomorphic for every $J \in \mathbb{S}_{A}$ with respect to the complex structures defined by left multiplication by $J$.

Proposition 4 implies that if $A$ is the algebra of quaternions or octonions, and $D$ intersects the real axis, then $f$ is slice regular on $\Omega_{D}$ if and only if it is Cullen regular in the sense introduced by Gentili and Struppa in [17, 18, 20, 16]. If $A$ is the real Clifford algebra $\mathbb{R}_{n}$, slice regularity generalizes the concept of slice monogenic functions introduced by Colombo, Sabadini and Struppa in [8]. If $f=$ $\mathcal{I}(F) \in \mathcal{S R}\left(\Omega_{D}\right), F \in C^{1}(D)$ and $D$ intersects the real axis, then the restriction of $f$ to the subspace of paravectors is a slice monogenic function. Conversely, every slice monogenic function is the restriction of a unique slice regular function.

\section{Product of slice functions}

In general, the pointwise product of two slice functions is not a slice function. However, pointwise product in the algebra $A_{\mathbb{C}}$ of $A$-stem functions induces a natural product on slice functions. 
Definition 9. Let $f=\mathcal{I}(F), g=\mathcal{I}(G) \in \mathcal{S}\left(\Omega_{D}\right)$. The product of $f$ and $g$ is the slice function $f \cdot g:=\mathcal{I}(F G) \in \mathcal{S}\left(\Omega_{D}\right)$.

The preceding definition is well-posed, since the pointwise product of $F$ and $G$ is complex intrinsic. The product is distributive and also associative if $A$ is an associative algebra. If the components $F_{1}, F_{2}$ of the first stem function $F$ are real-valued, then $(f \cdot g)(x)=f(x) g(x)$ for every $x \in \Omega_{D}$. In this case, we will use also the notation $f g$ in place of $f \cdot g$.

Definition 10. A slice function $f=\mathcal{I}(F)$ is called real if the $A$-valued components $F_{1}, F_{2}$ of its stem function are real-valued. Equivalently, $f$ is real if the spherical value $v_{s} f$ and the spherical derivative $\partial_{s} f$ are real-valued.

Real slice functions are characterized by the following property: for every $J \in \mathbb{S}_{A}$, the image $f\left(\mathbb{C}_{J} \cap \Omega_{D}\right)$ is contained in $\mathbb{C}_{J}$.

Let $f(x)=\sum_{j} x^{j} a_{j}$ and $g(x)=\sum_{k} x^{k} b_{k}$ be polynomials or convergent power series with coefficients $a_{j}, b_{k} \in A$. The usual product of polynomials, where $x$ is considered to be a commuting variable (cf. for example [27] and [14, 13]), can be extended to power series (cf. $[15,19]$ for the quaternionic case) by setting:

$$
(f * g)(x):=\sum_{n} x^{n}\left(\sum_{j+k=n} a_{j} b_{k}\right) .
$$

Proposition 5. Let $f$ and $g$ be polynomials or convergent power series. Then the product $f \cdot g$ coincides with the star product $f * g$, i.e. $\mathcal{I}(F G)=\mathcal{I}(F) * \mathcal{I}(G)$.

We now associate to every slice function the normal function, which is useful when dealing with zero sets. Our definition is equivalent to the symmetrization of quaternionic power series given in [15].

Definition 11. Let $f=\mathcal{I}(F) \in \mathcal{S}\left(\Omega_{D}\right)$. Then also $F^{c}(z):=F(z)^{c}=F_{1}(z)^{c}+$ $i F_{2}(z)^{c}$ is an $A$-stem function. We set $f^{c}:=\mathcal{I}\left(F^{c}\right), C N(F):=F F^{c}=n\left(F_{1}\right)-$ $n\left(F_{2}\right)+i t\left(F_{1} F_{2}{ }^{c}\right)$ and $N(f):=f \cdot f^{c}=\mathcal{I}(C N(F)) \in \mathcal{S}\left(\Omega_{D}\right)$. The slice function $N(f)$ will be called the normal function of $f$.

If $f$ is slice regular, then also $f^{c}$ and $N(f)$ are slice regular. If $A$ is the algebra of quaternions or octonions, then $C N(F)$ is complex-valued and then the normal function $N(f)$ is real. For a general algebra $A$, this is not true for every slice function. This is the motivation for the following definition.

Definition 12. A slice function $f=\mathcal{I}(F) \in \mathcal{S}\left(\Omega_{D}\right)$ is called admissible if $v_{s} f(x) \in$ $\mathcal{N}_{A}$ for every $x \in \Omega_{D}$ and the real vector subspace $\left\langle v_{s} f(x), \partial_{s} f(x)\right\rangle \subseteq \mathcal{N}_{A}$ for every $x \in \Omega_{D} \backslash \mathbb{R}$. Equivalently, $\left\langle F_{1}(z), F_{2}(z)\right\rangle \subseteq \mathcal{N}_{A}$ for every $z \in D$.

If $A=\mathbb{H}$ or $\mathbb{O}$, then every slice function is admissible. Moreover, if $f$ is real, then $f^{c}=f, N(f)=f^{2}$ and $f$ is admissible. If $f$ is admissible, then $C N(F)$ is complex-valued and then $N(f)$ is real.

Example 3. Consider the Clifford algebra $\mathbb{R}_{3}$ with the usual conjugation. Its normal cone contains the subspace $\mathbb{R}^{4}$ of paravectors. Every polynomial $p(x)=\sum_{n} x^{n} a_{n}$ with paravectors coefficients is admissible. The polynomial $p(x)=x e_{23}+e_{1}$ is an example of a non-admissible slice regular function. 
Theorem 6. Let $A$ be associative or $A=\mathbb{O}$. Then $N(f \cdot g)=N(f) N(g)$ for every admissible $f, g \in \mathcal{S}\left(\Omega_{D}\right)$.

Corollary 7. Let $A$ be associative or $A=\mathbb{O}$. Assume that $n(x)=n\left(x^{c}\right) \neq 0$ for every $x \in A \backslash\{0\}$ such that $n(x)$ is real. If $f$ and $g$ are admissible slice functions, then also the product $f \cdot g$ is admissible.

Example 4. Let $A=\mathbb{R}_{3}$. Consider the admissible polynomials $f(x)=x e_{2}+e_{1}$, $g(x)=x e_{3}+e_{2}$. Then $(f \cdot g)(x)=x^{2} e_{23}+x\left(e_{13}-1\right)+e_{12}$ is admissible, $N(f)=$ $N(g)=x^{2}+1$ and $N(f \cdot g)=\left(x^{2}+1\right)^{2}$.

\section{Zeros of slice functions}

The zero set $V(f)=\left\{x \in \Omega_{D} \mid f(x)=0\right\}$ of an admissible slice function $f$ has a particular structure. For every fixed $x \in \mathcal{Q}_{A}$, the "sphere" $\mathbb{S}_{x}$ is entirely contained in $V(f)$ or it contains at most one zero of $f$. Moreover, if $f$ is not real, there can be isolated, non-real zeros. These different types of zeros of a slice function correspond to the existence of zero-divisors in the complexified algebra $A_{\mathbb{C}}$.

Theorem 8 (Structure of $V(f)$ ). Let $f \in \mathcal{S}\left(\Omega_{D}\right)$ an admissible slice function. Let $x=\alpha+\beta J \in \Omega_{D}$ and $z=\alpha+i \beta \in D$. Then one of the following mutually exclusive statements holds:

(1) $\mathbb{S}_{x} \cap V(f)=\emptyset$.

(2) $\mathbb{S}_{x} \subseteq V(f)$. In this case $x$ is called a real (if $x \in \mathbb{R}$ ) or spherical (if $x \notin \mathbb{R}$ ) zero of $f$.

(3) $\mathbb{S}_{x} \cap V(f)$ consists of a single, non-real point. In this case $x$ is called an $\mathbb{S}_{A}$-isolated non-real zero of $f$.

Moreover, a real slice function has no $\mathbb{S}_{A}$-isolated non-real zeros, and for every admissible slice function $f$, we have $V(N(f))=\bigcup_{x \in V(f)} \mathbb{S}_{x}$.

Theorem 9. Let $\Omega_{D}$ be connected. If $f$ is slice regular and admissible on $\Omega_{D}$, and $N(f)$ does not vanish identically, then $\mathbb{C}_{J} \cap \bigcup_{x \in V(f)} \mathbb{S}_{x}$ is closed and discrete in $D_{J}=\mathbb{C}_{J} \cap \Omega_{D}$ for every $J \in \mathbb{S}_{A}$. If $\Omega_{D} \cap \mathbb{R} \neq \emptyset$, then $N(f) \equiv 0$ if and only if $f \equiv 0$.

In the quaternionic case, the structure theorem for the zero set of slice regular functions was proved by Pogorui and Shapiro [29] for polynomials and by Gentili and Stoppato [15] for power series.

Remark 2. If $\Omega_{D}$ does not intersect the real axis, a not identically zero slice regular function $f$ can have normal function $N(f) \equiv 0$. For example, let $J \in \mathbb{S}_{\mathbb{H}}$ be fixed. The admissible slice regular function defined on $\mathbb{H} \backslash \mathbb{R}$ by $f(x)=1-I J$ for $x \in \mathbb{C}_{I}^{+}=\{x=\alpha+\beta I \in A \mid \beta \geq 0\}$ has zero normal function.

Now we state a remainder theorem, which generalizes a result proved by Beck [1] for quaternionic polynomials and by Serôdio [33] for octonionic polynomials. 
Definition 13. For any $y \in \mathcal{Q}_{A}$, the characteristic polynomial of $y$ is the slice regular function on $\mathcal{Q}_{A}$

$$
\Delta_{y}(x):=N(x-y)=(x-y) \cdot\left(x-y^{c}\right)=x^{2}-x t(y)+n(y) .
$$

Proposition 10. The characteristic polynomial $\Delta_{y}$ of $y \in \mathcal{Q}_{A}$ is real. Two characteristic polynomials $\Delta_{y}$ and $\Delta_{y^{\prime}}$ coincides if and only if $\mathbb{S}_{y}=\mathbb{S}_{y^{\prime}}$. Moreover, $V\left(\Delta_{y}\right)=\mathbb{S}_{y}$

Theorem 11. Let $f \in \mathcal{S R}\left(\Omega_{D}\right)$ be an admissible slice regular function. Let $y \in$ $V(f)=\left\{x \in \mathcal{Q}_{A} \mid f(x)=0\right\}$. Then the following statements hold.

(1) If $y$ is a real zero, then there exists an admissible $g \in \mathcal{S R}\left(\Omega_{D}\right)$ such that $f(x)=(x-y) g(x)$.

(2) If $y \in \Omega_{D} \backslash \mathbb{R}$, then there exists an admissible $h \in \mathcal{S R}\left(\Omega_{D}\right)$ and $a, b \in A$ such that $\langle a, b\rangle \subseteq \mathcal{N}_{A}$ and $f(x)=\Delta_{y}(x) h(x)+x a+b$. Moreover,

- $y$ is a spherical zero of $f$ if and only if $a=b=0$.

- $y$ is an $\mathbb{S}_{A}$-isolated non-real zero of $f$ if and only if $a \neq 0$ (in this case $\left.y=-b a^{-1}\right)$.

If $f$ is real, then $g, h$ are real and $a=b=0$.

For every non-real $y \in V(f)$, the element $a \in \mathcal{N}_{A}$ which appears in the statement of the preceding theorem is the spherical derivative of $f$ at $x \in \mathbb{S}_{y}$.

Corollary 12. Let $f \in \mathcal{S R}\left(\Omega_{D}\right)$ be admissible. If $\mathbb{S}_{y}$ contains at least one zero of $f$, of whatever type, then $\Delta_{y}$ divides $N(f)$.

Definition 14. Let $f \in \mathcal{S R}\left(\Omega_{D}\right)$ be admissible, with $N(f) \not \equiv 0$. Given a nonnegative integer $s$ and an element $y$ of $V(f)$, we say that $y$ is a zero of $f$ of multiplicity $s$ if $\Delta_{y}^{s} \mid N(f)$ and $\Delta_{y}^{s+1} \nmid N(f)$. We will denote the integer $s$, called multiplicity of $y$, by $m_{f}(y)$.

In the case of $y$ real, the preceding condition is equivalent to $(x-y)^{s} \mid f$ and $(x-y)^{s+1} \nmid f$. If $y$ is a spherical zero, then $m_{f}(y) \geq 2$. In the case of quaternionic polynomials, the definition is equivalent to the one given in [2] and in [19].

Proposition 13. Let $A$ be associative. Let $f, g \in \mathcal{S}\left(\Omega_{D}\right)$. Then $V(f) \subseteq V(f \cdot g)$.

As shown in [33] for octonionic polynomials and in [23] for octonionic power series, if $A$ is not associative the statement of the proposition is no more true. However, we can still say something about the location of the zeros of $f \cdot g$ (cf. [23] for the precise relation linking the zeros of $f$ and $g$ to those of $f \cdot g$ ). For the associative case, see also $[27, \S 16]$.

Now we focus our attention on the zero set of slice regular admissible polynomials. If $p(x)=\sum_{j=0}^{m} x^{j} a_{j}$ is an admissible polynomial of degree $m$ with coefficients $a_{j} \in A$, then the normal polynomial

$$
N(p)(x)=\left(p * p^{c}\right)(x)=\sum_{n} x^{n}\left(\sum_{j+k=n} a_{j} a_{k}^{c}\right)
$$

has degree $2 m$ and real coefficients. A sufficient condition for the admissibility of $p$ is that the real vector subspace $\left\langle a_{0}, \ldots, a_{m}\right\rangle$ is contained in $\mathcal{N}_{A}$. 
Theorem 14 (Fundamental Theorem of Algebra with multiplicities). Let $p(x)=$ $\sum_{j=0}^{m} x^{j} a_{j}$ be an admissible polynomial of degree $m>0$ with coefficients in $A$. Then $V(p)=\left\{y \in \mathcal{Q}_{A} \mid p(y)=0\right\}$ is non-empty. More precisely, there are distinct "spheres" $\mathbb{S}_{x_{1}}, \ldots, \mathbb{S}_{x_{t}}$ such that

$$
V(p) \subseteq \bigcup_{k=1}^{t} \mathbb{S}_{x_{k}}=V(N(p)), \quad V(p) \cap \mathbb{S}_{x_{j}} \neq \emptyset \quad \text { for every } j,
$$

and, for any choice of zeros $y_{1} \in \mathbb{S}_{x_{1}}, \ldots, y_{t} \in \mathbb{S}_{x_{t}}$ of $p$, it holds $\sum_{k=1}^{t} m_{p}\left(y_{k}\right)=m$.

Remark 3. If $r$ denotes the number of real zeros of the polynomial $p, i$ the number of $\mathbb{S}_{A}$-isolated non-real zeros of $p$ and $s$ the number of "spheres" $\mathbb{S}_{y}(y \notin \mathbb{R})$ containing spherical zeros of $p$, we have that $r+i+2 s \leq \operatorname{deg}(p)$.

\section{Examples}

The following examples show the relevance of the quadratic cone and of admissibility for the algebraic and topological properties of the zero set of a polynomial.

(1) Every polynomial $\sum_{j=0}^{m} x^{j} a_{j}$, with paravector coefficients $a_{j}$ in the Clifford algebra $\mathbb{R}_{n}$ (with conjugation as antiinvolution), has $m$ roots (counted with their multiplicities) in the quadratic cone $\mathcal{Q}_{n}$. If the coefficients are real, then it has at least one root in the paravector space $\mathbb{R}^{n+1}$, since every "sphere" $\mathbb{S}_{y}$ intersect $\mathbb{R}^{n+1}$ (cf. [37, Theorem 3.1]).

(2) In $\mathbb{R}_{3}$, the admissible polynomial $p(x)=x^{2}+x e_{3}+e_{2}$ (cf. [37, Ex. 3]) has two isolated zeros

$$
y_{1}=\frac{1}{2}\left(1-e_{2}-e_{3}+e_{23}\right), y_{2}=\frac{1}{2}\left(-1+e_{2}-e_{3}+e_{23}\right)
$$

in $\mathcal{Q}_{3} \backslash \mathbb{R}^{4}$. They can be computed by solving the complex equation $C N(P)=$ $z^{4}+z^{2}+1=0$ to find the two "spheres" $\mathbb{S}_{y_{1}}, \mathbb{S}_{y_{2}}$ and then using the Remainder Theorem (Theorem 11) with $\Delta_{y_{1}}=x^{2}-x+1$ and $\Delta_{y_{2}}=x^{2}+x+1$.

(3) In $\mathbb{R}_{3}$, the polynomial $p(x)=x e_{23}+e_{1}$ vanishes only at $y=e_{123} \notin \mathcal{Q}_{3}$. Note that $p$ is not admissible: $e_{1}, e_{23} \in \mathcal{N}_{3}$, but $e_{1}+e_{23} \notin \mathcal{N}_{3}$.

(4) An admissible polynomial of degree $m$, even in the case of non-spherical zeros, can have more than $m$ roots in the whole algebra. For example, $p(x)=x^{2}-1$ has four roots in $\mathbb{R}_{3}$, two in the quadratic cone $(x= \pm 1)$ and two outside it $\left(x= \pm e_{123}\right)$.

(5) Outside the quadratic cone, an admissible polynomial can have infinite non-spherical zeros. For example, in $\mathbb{R}_{1,2}$ the polynomial $p(x)=x^{2}-1$ has zeros \pm 1 in $\mathcal{Q}_{1,2}$, while in $\mathbb{R}_{1,2} \backslash \mathcal{Q}_{1,2}$ it vanishes on the 4 -dimensional set

$\left\{x \mid x_{0}=x_{123}=0, x_{2} x_{13}-x_{1} x_{23}-x_{3} x_{12}=0, x_{1}^{2}+x_{12}^{2}+x_{13}^{2}-x_{2}^{2}-x_{3}^{2}-x_{23}^{2}=1\right\}$.

(6) In $\mathbb{R}_{3}$, the admissible polynomial $p(x)=x^{3}-1$ has zero set $V(p)=\{1\} \cup \mathbb{S}_{y}$ $\left(y=-\frac{1}{2}+\frac{\sqrt{3}}{2} J, J \in \mathbb{S}_{\mathbb{R}_{3}}\right)$ in $\mathcal{Q}_{3}$, while in $\mathbb{R}_{3} \backslash \mathcal{Q}_{3}$ the polynomial $p$ vanishes on 
the two 2-spheres

$S_{ \pm}=\left\{x=\frac{1}{4}+x_{1}\left(e_{1} \pm e_{23}\right)+x_{2}\left(e_{2} \pm e_{13}\right)+x_{3}\left(e_{3} \pm e_{12}\right) \pm \frac{3}{4} e_{123} \mid x_{1}^{2}+x_{2}^{2}+x_{3}^{2}=\frac{3}{16}\right\}$.

(7) In the algebras $\mathbb{H}, \mathbb{O}, \mathbb{R}_{3}$, the solutions of the equation $x^{2}=-1$ are exactly the elements of $\mathbb{S}_{\mathbb{R}_{3}}$, i.e. they all belong to the quadratic cone. This is not the case for other algebras. In $\mathbb{R}_{1,2}, e_{123}^{2}=-1$, but $e_{123} \notin \mathcal{Q}_{1,2}$. In $\mathbb{R}_{4}$, the equation $x^{2}=-1$ has many solutions: the square roots of -1 in the "sphere" $\mathbb{S}_{\mathbb{R}_{4}}$, but also infinite other points outside the quadratic cone:

$$
x=\sum_{i=1}^{4} x_{i} e_{i}+x_{1234} e_{1234}, \text { with } \sum_{i=1}^{4} x_{i}^{2}-x_{1234}^{2}=1 .
$$

(8) The admissibility of a slice function depends on the algebra. For example, the polynomial $p(x)=x^{2}+x e_{1}+2$ is admissible on the algebra $\mathbb{R}_{3}$ (w.r.t. conjugation), where its zero set is the union of $V(p)=\left\{e_{1},-2 e_{1}\right\} \subseteq \mathcal{Q}_{3}$ and the subset $\left\{-\frac{1}{2} e_{1} \pm \frac{3}{2} e_{23}\right\}$ of $\mathbb{R}_{3} \backslash \mathcal{Q}_{3}$. The same polynomial is not admissible on the algebra $\mathbb{R}_{1,2}$, and it has no zeros in $\mathcal{Q}_{1,2}$.

(9) The admissibility depends also on the antiinvolution chosen in the algebra. For example, in the algebra $\mathbb{R}_{n}$ with the reversion as fixed antiinvolution, a polynomial with paravector coefficient can be not admissible. For $n=2, p(x)=x e_{1}+1$ is not admissible, since $\left\langle 1, e_{1}\right\rangle \nsubseteq \mathcal{N}_{2}^{*}$. This property reflects in the fact that the unique zero of $p$ in $\mathbb{R}_{2}$ does not belong to the quadratic cone $\mathcal{Q}_{2}^{*}=\left\{x \in \mathbb{R}_{2} \mid x_{1}=x_{2}=0\right\}$. The same holds in every $\mathbb{R}_{n}$. Instead, the polynomials with coefficients in the real vector subspace of 1 -vectors are admissible in $\mathbb{R}_{n}$ (w.r.t. reversion).

(10) The reality of $N(p)$ is not sufficient to get the admissibility of $p$. For example, in $\mathbb{R}_{3}$, the polynomial $p(x)=x^{2} e_{123}+x\left(e_{1}+e_{23}\right)+1$ has real normal function $N(p)=\left(x^{2}+1\right)^{2}$, but the spherical derivative $\partial_{s} p=t(x) e_{123}+e_{1}+e_{23}$ has $N\left(\partial_{s} p\right)$ not real. In particular, $\partial_{s} p(J)=e_{1}+e_{23} \notin \mathcal{N}_{3}$ for every $J \in \mathbb{S}_{\mathbb{R}_{3}}$. The non-admissibility of $p$ is reflected by the existence of a $S^{1}$ of distinct zeros on $\mathbb{S}_{\mathbb{R}_{3}}$, where $p$ does not vanish identically.

\section{References}

[1] B. Beck. Sur les équations polynomiales dans les quaternions. Enseign. Math. (2), 25(3-4):193-201 (1980), 1979.

[2] U. Bray and G. Whaples. Polynomials with coefficients from a division ring. Canad. J. Math., 35(3):509-515, 1983.

[3] F. Colombo, G. Gentili, and I. Sabadini. A Cauchy kernel for slice regular functions. Ann. Global Anal. Geom., 37:361-378, 2010.

[4] F. Colombo, G. Gentili, I. Sabadini, and D. C. Struppa. Extension results for slice regular functions of a quaternionic variable. Adv. Math., 222(5):1793-1808, 2009.

[5] F. Colombo and I. Sabadini. A structure formula for slice monogenic functions and some of its consequences. In Hypercomplex analysis, Trends Math., pages 101-114. Birkhäuser, Basel, 2009. 
[6] F. Colombo and I. Sabadini. The Cauchy formula with s-monogenic kernel and a functional calculus for noncommuting operators. J. Math. Anal. Appl., 373:655-679, 2011.

[7] F. Colombo, I. Sabadini, F. Sommen, and D. C. Struppa. Analysis of Dirac systems and computational algebra, volume 39 of Progress in Mathematical Physics. Birkhäuser Boston Inc., Boston, MA, 2004.

[8] F. Colombo, I. Sabadini, and D. C. Struppa. Slice monogenic functions. Israel J. Math., 171:385-403, 2009.

[9] F. Colombo, I. Sabadini, and D. C. Struppa. An extension theorem for slice monogenic functions and some of its consequences. Israel J. Math., 177:369-389, 2010.

[10] S. Eilenberg and I. Niven. The "fundamental theorem of algebra" for quaternions. Bull. Amer. Math. Soc., 50:246-248, 1944.

[11] S. Eilenberg and N. Steenrod. Foundations of algebraic topology. Princeton University Press, Princeton, New Jersey, 1952.

[12] R. Fueter. Die Funktionentheorie der Differentialgleichungen $\Delta u=0$ und $\Delta \Delta u=0$ mit vier reellen Variablen. Comment. Math. Helv., 7(1):307-330, 1934.

[13] I. Gelfand, S. Gelfand, V. Retakh, and R. L. Wilson. Factorizations of polynomials over noncommutative algebras and sufficient sets of edges in directed graphs. Lett. Math. Phys., 74(2):153-167, 2005.

[14] I. Gelfand, V. Retakh, and R. L. Wilson. Quadratic linear algebras associated with factorizations of noncommutative polynomials and noncommutative differential polynomials. Selecta Math. (N.S.), 7(4):493-523, 2001.

[15] G. Gentili and C. Stoppato. Zeros of regular functions and polynomials of a quaternionic variable. Michigan Math. J., 56(3):655-667, 2008.

[16] G. Gentili, C. Stoppato, D. C. Struppa, and F. Vlacci. Recent developments for regular functions of a hypercomplex variable. In Hypercomplex analysis, Trends Math., pages 165-186. Birkhäuser, Basel, 2009.

[17] G. Gentili and D. C. Struppa. A new approach to Cullen-regular functions of a quaternionic variable. C. R. Math. Acad. Sci. Paris, 342(10):741-744, 2006.

[18] G. Gentili and D. C. Struppa. A new theory of regular functions of a quaternionic variable. Adv. Math., 216(1):279-301, 2007.

[19] G. Gentili and D. C. Struppa. On the multiplicity of zeroes of polynomials with quaternionic coefficients. Milan J. Math., 76:15-25, 2008.

[20] G. Gentili and D. C. Struppa. Regular functions on the space of Cayley numbers. Rocky Mountain J. Math., 40(1):225-241, 2010.

[21] G. Gentili, D. C. Struppa, and F. Vlacci. The fundamental theorem of algebra for Hamilton and Cayley numbers. Math. Z., 259(4):895-902, 2008.

[22] R. Ghiloni and A. Perotti. Slice regular functions on real alternative algebras. $A d v$. Math. DOI: 10.1016/j.aim.2010.08.015, 2010.

[23] R. Ghiloni and A. Perotti. Zeros of regular functions of quaternionic and octonionic variable: a division lemma and the camshaft effect. To appear in Ann. Mat. Pura Appl. DOI: 10.1007/s10231-010-0162-1.

[24] B. Gordon and T. S. Motzkin. On the zeros of polynomials over division rings. Trans. Amer. Math. Soc., 116:218-226, 1965. 
[25] K. Gürlebeck, K. Habetha, and W. Sprößig. Holomorphic functions in the plane and $n$-dimensional space. Birkhäuser Verlag, Basel, 2008.

[26] Y.-L. Jou. The "fundamental theorem of algebra" for Cayley numbers. Acad. Sinica Science Record, 3:29-33, 1950.

[27] T. Y. Lam. A first course in noncommutative rings, volume 131 of Graduate Texts in Mathematics. Springer-Verlag, New York, 1991.

[28] I. Niven. Equations in quaternions. Amer. Math. Monthly, 48:654-661, 1941.

[29] A. Pogorui and M. Shapiro. On the structure of the set of zeros of quaternionic polynomials. Complex Var. Theory Appl., 49(6):379-389, 2004.

[30] T. Qian. Generalization of Fueter's result to $\mathbf{R}^{n+1}$. Atti Accad. Naz. Lincei Cl. Sci. Fis. Mat. Natur. Rend. Lincei (9) Mat. Appl., 8(2):111-117, 1997.

[31] H. Rodríguez-Ordóñez. A note on the fundamental theorem of algebra for the octonions. Expo. Math., 25(4):355-361, 2007.

[32] M. Sce. Osservazioni sulle serie di potenze nei moduli quadratici. Atti Accad. Naz. Lincei. Rend. Cl. Sci. Fis. Mat. Nat. (8), 23:220-225, 1957.

[33] R. Serôdio. On octonionic polynomials. Adv. Appl. Clifford Algebr., 17(2):245-258, 2007.

[34] F. Sommen. On a generalization of Fueter's theorem. Z. Anal. Anwendungen, 19(4):899-902, 2000.

[35] A. Sudbery. Quaternionic analysis. Math. Proc. Cambridge Philos. Soc., 85(2):199224, 1979.

[36] N. Topuridze. On the roots of polynomials over division algebras. Georgian Math. J., 10(4):745-762, 2003.

[37] Y. Yang and T. Qian. On sets of zeroes of Clifford algebra-valued polynomials. Acta Math. Sci. Ser. B Engl. Ed., 30(3):1004-1012, 2010.

Riccardo Ghiloni

Department of Mathematics

University of Trento

Via Sommarive 14

I-38123 Povo-Trento

ITALY

e-mail: ghiloni@science. unitn.it

Alessandro Perotti

Department of Mathematics

University of Trento

Via Sommarive 14

I-38123 Povo-Trento

ITALY

e-mail: perotti@science.unitn.it 